Pacific Journal of Mathematics

ON THE REFLECTION OF HARMONIC FUNCTIONS AND OF 


\section{ON THE REFLECTION OF HARMONIC FUNCTIONS AND OF SOLUTIONS OF THE WAVE EQUATION}

\section{VLADIMIR FILIPPENKO}

Introduction. While the analytic extension of a harmonic function across analytic differential boundary conditions is always possible for the case of two independent variables [3], no comparable global theorem exists for harmonic functions in $N>2$ variables.

This work is concerned with the problem of global extension of a harmonic function $U(x, y, z)$ across a plane on which $U$ satisfies a linear differential boundary condition of the form

$$
B(U) \equiv \frac{\partial U}{\delta z}+P_{n}(x, y) U=0 \quad \text { on } \sigma(z=0),
$$

where $P_{n}(x, y)$ is a polynomial of degree $n$. It is assumed here that the given function $U$ is $C^{1}$ in the closure of a cylindrical domain $R:\left\{x^{2}+y^{2}<\rho^{2},-l<z<0\right\}$.

The possibility of harmonic reflection is obvious for $n=0, P_{n}=$ const. as $B(U)$ itself is harmonic. Since it vanishes on $z=0$, it can be extended harmonically, and the harmonic extension of $U$ can then be found by integrating with respect to $z$. But such procedure is no longer available in our case. We shall show, how our problem can be reduced to that of solving an initial value problem of a certain hyperbolic differential equation (1.22) of order $2 n$ with distinct characteristic surfaces (of normal type).

Classical considerations yield the analyticity of $U$ on $\sigma$ and, therefore, its harmonic extensibility across $\sigma$ into a neighborhood of $\sigma$. Our result asserts that this neighborhood is the whole of the mirror image of $R$, denoted by $\bar{R}$.

Our method consists of constructing a new function $V(x, y, z)$ from $U$ and a differential expression in $V$ (see (1.6) and (1.18)), which is harmonic in $R$ and vanishes on $z=0$. Thus, this expression in $V$ can be first extended into $R \cup \sigma \cup \bar{R}$ as a harmonic function $\varphi(x, y, z)$. The solution of the differential equation thus obtained for $V$ in $\bar{R}$ is impeded by its degeneracy. To remove this degeneracy we add to the differential equation the Laplacian of $V$ and its higher derivatives in such a way as to obtain a normal hyperbolic problem (1.22), whose solution is guaranteed by a result of I. G. Petrovsky. This modification of the differential equation can be done in infinitely many ways, in particular, so as to make the characteristic surfaces close down on

Received February 20, 1964. This work was supported by the Office of Naval Research, \$222 (62) 
parallels to the $z$-axis. Local extensibility of $U$, together with the solution of the modified equation, then yields the global extension of $U$. We note, that this method works equally well for $N>3$ independent variables.

The above described method, however, seems to fail in the case of the wave equation when $\sigma$ is part of the tilmelike plane $z=0$, and the boundary condition on it is as simple as $U_{z}+x U=0$.

On the other hand, the oblique derivative problem for the wave equation $U_{x x}+U_{y y}-U_{t t}=0$, whose solution satisfies the boundary condition

$$
B^{\prime}(U) \equiv U_{x}+\alpha U_{y}+(A y+B) U=0 \quad \text { on } x=0,
$$

yields to a similarly motivated, yet formally different attack. The domain of extension in this case depends on $\alpha \neq 0$.

I would like to take this opportunity to express my gratitude to professor H. Lewy who suggested this problem and offered advice during its investigation.

1. Analytic extension of harmonic functions. We consider an open cylindrical domain $R:\left\{x^{2}+y^{2}<\rho^{2},-l<z<0\right\}$ and the plane region $\sigma:\left\{x^{2}+y^{2}<\rho^{2}, z=0\right\}$. Denote by $\bar{R}$ the mirror image of $R$ with respect to the $z=0$ plane.

Let there be given a real function $U(x, y, z), U \in C^{1}$ in the closure of $R$, such that:

$$
\begin{array}{cc}
U_{x x}+U_{y y}+U_{z z} \equiv \Delta U=0 & \text { in } R \\
\frac{\partial U}{\partial z}+P_{n}(x, y) U=0 & \text { on } \sigma
\end{array}
$$

where $P_{n}(x, y)$ is a polynomial in $x, y$ of degree $n$, given in the form

$$
P_{n}(x, y)=\sum_{k=0}^{n} \sum_{m=0}^{k} A_{k m} x^{k-m} y^{m},
$$

the coefficients $A_{k m}$ being real.

LEMmA 1. If $U(x, y, z)$ is harmonic in $R, U \in C^{1}$ in $R \cup \partial R$, and satisfies condition (1.2) on $\sigma$, then $U$ can be harmonically extended into $R \cup \sigma \cup G$, where $G$ is the portion $z>0$ of some neighborhood of $\sigma$.

Proof. Since $U$ is $C^{1}$ in $R \cup \partial R$, we have by Green's formula

$$
4 \pi U(X)=\iint_{\partial R}\left\{\frac{1}{|X-\tau|} \frac{\partial U(\tau)}{\partial n}-U(\tau) \frac{\partial}{\partial n} \frac{1}{|X-\tau|}\right\} d \tau
$$


where $X=(x, y, z), \tau=(\xi, \eta, \zeta), n$ is the outer normal, and integration is over the surface of the cylinder $\xi^{2}+\eta^{2}=\rho^{2}, \zeta=-l, \zeta=0$. By (1.2) this becomes

$$
4 \pi U(X)=A(X)-\iint_{\sigma}\left\{\frac{P_{n}(\tau) U(\tau)}{|X-\tau|}+U(\tau) \frac{\partial}{\partial \zeta} \frac{1}{|X-\tau|}\right\} d \tau
$$

where $A(X)$ stands for the integral in (1.4) taken over the lateral surface and the lower base of the cylinder. By passage to the limit as $X$ tends to $X^{\prime} \in \sigma$, one obtains in a manner familiar in potential theory,

$$
2 \pi U\left(X^{\prime}\right)=A\left(X^{\prime}\right)-\iint_{\sigma} \frac{P_{n}\left(\tau^{\prime}\right) U\left(\tau^{\prime}\right)}{\left|X^{\prime}-\tau^{\prime}\right|} d \tau^{\prime}
$$

where $A\left(X^{\prime}\right)$ is an analytic function on $\sigma$. This integral equation is an especially simple case of E. Hopf's equation (6.1) ([2], page 220), and his method yields immediately the result, that $U(x, y, 0)$ is analytic on the open disc $\sigma$.

Since, due to condition (1.2), $U_{z}(x, y, 0)$ is also analytic, we obtain from the Cauchy-Kowalewski theorem, that there exists an analytic solution $\widetilde{U}$ of Cauchy's problem with $\widetilde{U}=U, \widetilde{U}_{z}=U_{z}$ on $\sigma$ for $\Delta \widetilde{U}=$ 0 in some neighborhood $G$ of $\sigma$.

If we continue $U$, given in $R \cup \sigma$, as $\widetilde{U}$ in $G-R-\sigma$, this new function is, according to well known arguments, harmonic in $R \cup \sigma \cup G$.

We now introduce the symbolic notation

$$
D_{z}^{-1} f(x, y, z)=\int_{0}^{z} f(x, y, \zeta) d \zeta,
$$

and define an analytic function $V(x, y, z)$ for $(x, y, z) \in R \cup \sigma$ :

$$
V(x, y, z) \equiv D_{z}^{-(2 n-1)} U(x, y, z)+\sum_{k=0}^{2 n-2} \frac{z^{k}}{k !} F_{k}(x, y),
$$

where the functions $F_{k}(x, y)(0 \leqq k \leqq 2 n-2)$ are solutions of the following equations on $\sigma$ :

$$
\begin{aligned}
& \left(\frac{\partial^{2}}{\partial x^{2}}+\frac{\partial^{2}}{\partial y^{2}}\right) F_{2 n-2}+U_{z}(x, y, 0)=0 \\
& \left(\frac{\partial^{2}}{\partial x^{2}}+\frac{\partial^{2}}{\partial y^{2}}\right) F_{2 n-3}+U(x, y, 0)=0 \\
& \left(\frac{\partial^{2}}{\partial x^{2}}+\frac{\partial^{2}}{\partial y^{2}}\right) F_{r}+F_{r+2}=0 \quad(0 \leqq r \leqq 2 n-4)
\end{aligned}
$$

with, say, boundary values zero on $x^{2}+y^{2}=\rho^{2}$.

The choice of these functions is motivated by the requirements 


$$
\Delta V=0 \quad \text { in } R
$$

$$
V_{z^{2 n}}+P_{n}(x, y) V_{z^{2 n-1}}=0 \quad \text { on } \sigma,
$$

which are easily verified.

Let $s$ stand for either $x$ or for $y$, and denote

$$
H_{s, z}=s \frac{\partial}{\partial z}-z \frac{\partial}{\partial s}, \quad H_{s, 0}^{m}=\left.\left(H_{s, z}\right)^{m}\right|_{z=0} \text {. }
$$

We then have the identities:

$$
\begin{aligned}
H_{s, 0}^{2 m+1} & =\sum_{k=0}^{m} \sum_{j=0}^{k} a_{j k}^{m} s^{2 k-j+1} \frac{\partial^{2 k-j+1}}{\partial s^{j} \partial z^{2 k-2 j+1}} & (m=0,1,2, \cdots) \\
H_{s, 0}^{2 m} & =\sum_{k=0}^{m} \sum_{j=0}^{k} b_{j k}^{m} s^{2 k-j} \frac{\partial^{2 k-j}}{\partial s^{j} \partial z^{2 k-2 j}} & (m=1,2, \cdots)
\end{aligned}
$$

where the coefficients $a_{j k}^{m}$ and $b_{j k}^{m}$ are real numbers, and $a_{0 m}^{m}=b_{0 m}^{m}=1 . \mathrm{g}$

Proof. Introducing new variables $t=s+i z, \tau=s-i z$, we may write, with $\partial / \partial t=1 / 2[(\partial / \partial s)-i(\partial / \partial z)]$ and $\partial / \partial \tau=1 / 2[(\partial / \partial s)+i(\partial / \partial z)]$

$$
H_{s, z}=i\left(t \frac{\partial}{\partial t}-\tau \frac{\partial}{\partial \tau}\right) \text {. }
$$

Hence,

$$
H_{s, 0}^{n}=\left.i^{n} \sum_{p=0}^{n}(-1)^{p}\left(\begin{array}{l}
n \\
p
\end{array}\right)\left(t \frac{\partial}{\partial t}\right)^{n-p}\left(\tau \frac{\partial}{\partial \tau}\right)^{p}\right|_{z=0} \cdot
$$

Now, for any variable $\xi$ (real or complex)

$$
\left(\xi \frac{\partial}{\partial \xi}\right)^{r}=\sum_{h=0}^{r} B_{h}^{r} \xi^{h} \frac{\partial^{h}}{\partial \xi^{h}}
$$

where the coefficients $B_{h}^{r}$ are nonnegative integers. Since $\partial \tau / \partial t=\partial t / \partial \tau=$ 0 , and for $z=0$ we have $t=\tau=s$, each term in (1.14) is, but for a constant coefficient, of the form

$$
\left.t^{\alpha} \tau^{\beta} \frac{\partial^{\alpha+\beta}}{\partial t^{\alpha} \partial \tau^{\beta}}\right|_{z=0}=s^{\alpha+\beta} \frac{\partial^{\alpha+\beta}}{\partial t^{\alpha} \partial \tau^{\beta}} \quad(1 \leqq \alpha+\beta \leqq n) .
$$

Since $\partial^{2} / \partial t \partial \tau=1 / 4\left[\left(\partial^{2} / \partial s^{2}\right)+\left(\partial^{2} / \partial z^{2}\right)\right]$, each term in (1.14) is, but for a constant coefficient, either of the form

$$
s^{\alpha+\beta}\left[\frac{\partial^{2}}{\partial s^{2}}+\frac{\partial^{2}}{\partial z^{2}}\right]^{\min \alpha, \beta}\left(\frac{\partial}{\partial t}\right)^{|\alpha-\beta|},
$$

or of the form

$$
\boldsymbol{s}^{\alpha+\beta}\left[\frac{\partial^{2}}{\partial s^{2}}+\frac{\partial^{2}}{\partial z^{2}}\right]^{\min \alpha, \beta}\left(\frac{\partial}{\partial \tau}\right)^{|\alpha-\beta|}
$$


Now, for any positive integer $q,[(\partial / \partial s) \pm i(\partial / \partial z)]^{q}$ has terms with imaginary coefficients only of the form $\partial^{\lambda+\mu} / \partial s^{\lambda} \partial z^{\mu}$, where $\mu$ is odd, and terms with real coefficients only of the form $\partial^{\lambda+\mu} / \partial s^{\lambda} \partial z^{\mu}$, where $\mu$ is even $(\lambda+\mu=q)$. Consequently, as $H_{s 0}^{n}$ must have real coefficients, it will consist of terms $s^{\lambda+\mu}\left(\partial^{\lambda+\mu} / \partial s^{\lambda} \partial z^{\mu}\right)$, where $\mu$ is odd when $n$ is odd, and $\mu$ is even when $n$ is even, which implies identities (1.12) and (1.13).

LEMma 2. There exist differential operators

$$
D_{i}^{r} \equiv \sum_{h=0}^{r} C_{i r h} \frac{\partial^{r}}{\partial s^{h} \partial z^{r-h}},
$$

where $C_{i r h}$ are real constants, such that

$$
s^{p} \frac{\partial^{2 p-1}}{\partial z^{2 p-1}}=\sum_{i=1}^{P} H_{s, 0}^{i} D_{i}^{p-1} \quad \text { for } z=0
$$

Proof. Starting from the definition of $H_{s 0}^{n}$ we see, that the above statement holds for $p=1$ and $p=2$, with $D_{1}^{0}=1, D_{1}^{1}=\partial / \partial s$ and $D_{2}^{1}=$ $\partial / \partial z$. Assuming, that the statement holds for $p \leqq 2 n$, we prove by induction, that it also holds for $p=2 n+1$ and $p=2 n+2$.

Since, by assumption, the lemma holds for $p \leqq 2 n$, we have for any nonnegative integers $\alpha$ and $\beta$, and any positive integer $q \leqq 2 n$

$$
s^{q} \frac{\partial^{2 q-1+\alpha+\beta}}{\partial s^{\alpha} \partial z^{2 q-1+\beta}}=\sum_{i=1}^{q} H_{s, 0}^{i} D_{i}^{q-1+\alpha+\beta} .
$$

But identity (1.12) yields

$$
\begin{aligned}
s^{2 n+1} \frac{\partial^{4 n+1}}{\partial z^{4 n+1}}= & H_{s, 0}^{2 n+1} \frac{\partial^{2 n}}{\partial z^{2 n}}-\sum_{j=1}^{n} a_{j n}^{n} s^{2 n-j+1} \frac{\partial^{4 n-j+1}}{\partial s^{j} \partial z^{4 n-2 j+1}} \\
& -\sum_{k=0}^{n-1} \sum_{j=0}^{k} a_{j k}^{n} s^{2 k-j+1} \frac{\partial^{2 n+2 k-j+1}}{\partial s^{j} \partial z^{2 n+2 k-2 m+1}} .
\end{aligned}
$$

We now observe, that all terms on the right hand side of the above expression are of the form (1.16), where $q=2 n-j+1 \quad(1 \leqq j \leqq n$, i.e. $q \leqq 2 n), \alpha=j, \beta=0$, for terms contained in the simple sum, and $q=2 k-j+1 \quad(0 \leqq j \leqq k, 0 \leqq k \leqq n-1$, i.e. $q \leqq 2 n-1), \alpha=j$, $\beta=2 n-2 k$, for terms contained in the double sum. Hence, the above lemma holds for $p=2 n+1$.

A similar argument, which utilizes identity (1.13) instead of (1.12), shows that this lemma holds also for $p=2 n+2$, and thus completes the proof.

We now introduce the differential operator of order $2 p-1$

$$
Q_{s, z}^{p} \equiv \sum_{i=1}^{p} H_{s, z}^{i} D_{i}^{p-1} \quad(p \geqq 1)
$$


where the $D_{i}^{p-1}$ are those of (1.15). Note that, for $z=0, Q_{s, 0}^{p}=$ $\boldsymbol{s}^{p}\left(\partial^{2 p-1} / \partial z^{2 p-1}\right)$.

Define an analytic function $\varphi(x, y, z)$ for $(x, y, z) \in R \cup \sigma$ :

$$
\varphi(x, y, z) \equiv V_{z^{2 n}}(x, y, z)+N V(x, y, z) .
$$

Here $V(x, y, z)$ is the function defined in (1.6), and $N=N(x, y, z)$ is a differential operator of order $2 n-1$ defined by:

$$
\begin{aligned}
N(x, y, z)= & A_{00} \frac{\partial^{2 n-1}}{\partial z^{2 n-1}}+\sum_{k=1}^{n}\left(A_{k 0} Q_{x, z}^{k}+A_{k k} Q_{y, z}^{k}\right) \frac{\partial^{2 n-2 k}}{\partial z^{2 n-2 k}} \\
& +\sum_{k=2}^{n} \sum_{m=1}^{k-1} A_{k m} Q_{x, z}^{k-m} Q_{y, z}^{m} \frac{\partial^{2 n-2 k+1}}{\partial z^{2 n-2 k+1}}
\end{aligned}
$$

where the coefficients $A_{k m}$ are the coefficients of the polynomial $P_{n}(x, y)$ defined in (1.3).

Lemma 3. $\Delta \varphi=0$ in $R$, and $\varphi(x, y, 0)=0$.

Proof. Note, that $\Delta H_{x, z}=H_{x, z} \Delta$ and $\Delta H_{y, z}=H_{y, z} \Delta$. Thus, by (1.17) and (1.19), the operators $\Delta$ and $N$ commute. Therefore, operating on both sides of (1.18) by $\Delta$, and making use of (1.10), we obtain

$$
\Delta \varphi=\left(\frac{\partial^{2 n}}{\partial z^{2 n}}+N\right) \Delta V=0 \quad \text { in } R .
$$

Making use of (1.17) and (1.15) we may write, for $z=0$,

$$
\begin{aligned}
& \left.N(x, y, z) V(x, y, z)\right|_{z=0} \\
& =\left\{A_{00} \frac{\partial^{2 n-1}}{\partial z^{2 n-1}}+\sum_{k=1}^{n}\left(A_{k 0} x^{k} \frac{\partial^{2 k-1}}{\partial z^{2 k-1}}+A_{k k} y^{k} \frac{\partial^{2 k-1}}{\partial z^{2 k-1}}\right) \frac{\partial^{2 n-2 k}}{\partial z^{2 n-2 k}}\right. \\
& \left.\quad+\sum_{k=2}^{n} \sum_{m=1}^{k-1} A_{k m} x^{k-m} \frac{\partial^{2 k-2 m-1}}{\partial z^{2 k-2 m-1}} y^{m} \frac{\partial^{2 m-1}}{\partial z^{2 m-1}} \frac{\partial^{2 n-2 k+1}}{\partial z^{2 n-2 k+1}}\right\}\left.V(x, y, z)\right|_{z=0},
\end{aligned}
$$

which becomes

$$
\left.N(x, y, z) V(x, y, z)\right|_{z=0}=\sum_{k=0}^{n} \sum_{m=0}^{k} A_{k m} x^{k-m} y^{m} V_{z^{2 n-1}}(x, y, 0) .
$$

Thus, setting $z=0$ in (1.18) and making use of (1.20) and (1.11) we obtain $\varphi(x, y, 0)=0$.

Hence, if we set for $(x, y, z) \in \bar{R} \cup \sigma$

$$
\varphi(x, y, z)=-\varphi(x, y,-z) \equiv-\left.\left[\frac{\partial^{2 n}}{\partial \zeta^{2 n}}+N(x, y, \zeta)\right] V(x, y, \zeta)\right|_{\zeta=-z},
$$

then $\varphi$ is harmonic in $R \cup \sigma \cup \vec{R}$.

Since $\varphi(x, y,-z)$ is known for $(x, y, z) \in \bar{R} \cup \sigma$, we shall seek a function $\bar{V}(x, y, z)$ for $(x, y, z) \in \bar{R} \cup \sigma$, which satisfies the following overdetermined system (S) for $\bar{V}$ on $z>0$ : 


$$
\left.\begin{array}{c}
\bar{V}_{z^{2 n}}(x, y, z)+N(x, y, z) \bar{V}(x, y, z)=-\varphi(x, y,-z) \\
\Delta \bar{V}(x, y, z)=0 \\
\left.\frac{\partial^{r} \bar{V}}{\partial z^{r}}\right|_{z=0}=F_{r}(x, y) \quad 0 \leqq r \leqq 2 n-2 \quad \bar{V}_{z^{2 n-1}}(x, y, 0)=U(x, y, 0)
\end{array}\right\}
$$

where the functions $F_{r}(x, y)$ are defined by the equations (1.7), (1.8) and (1.9).

Since, by Lemma $1, U$ can be continued into $R \cup \sigma \cup G$ as an analytic function, the formula (1.6) can be used to define a function $V^{*}(x, y, z)$ as an analytic function in $R \cup \sigma \cup G^{\prime}$, where $G^{\prime}$ consists of all those points of $G$, which can be joined in $G$ to points of $\sigma$ by parallels to the $z$-axis. This, so defined function $V^{*}$ is harmonic in $R \cup \sigma \cup G^{\prime}$, satisfies the initial conditions of (S), and

$$
\begin{aligned}
V_{z^{2 n}}^{*}(x, y, z)+N V^{*} & =-\left.\left[\frac{\partial^{2 n}}{\partial \zeta^{2 n}}+N(x, y, \zeta)\right] V^{*}(x, y, \zeta)\right|_{\zeta=-z} \\
& =-\varphi(x, y,-z) \quad \text { in } G^{\prime} .
\end{aligned}
$$

Thus, a solution $V^{*}(x, y, z)$ of system (S) exists for $(x, y, z) \in G^{\prime} \cup \sigma$.

To investigate the size of the domain into which $V(x, y, z)$ can be continued, consider the solution of the following Cauchy problem:

$$
\begin{aligned}
M \bar{V}(x, y, z) & \equiv \prod_{i=1}^{n}\left[\frac{\partial^{2}}{\partial z^{2}}-\alpha_{i}\left(\frac{\partial^{2}}{\partial x^{2}}+\frac{\partial^{2}}{\partial y^{2}}\right)\right] \bar{V}+\beta N(x, y, z) \bar{V} \\
& =-\beta \varphi(x, y,-z)
\end{aligned}
$$

$$
\left.\frac{\partial^{r} \bar{V}}{\partial z^{r}}\right|_{z=0}=F_{r}(x, y)(0 \leqq r \leqq 2 n-2), \bar{V}_{z^{2 n-1}}(x, y, 0)=U(x, y, 0)
$$

where $\alpha_{i}(i=1,2, \cdots, n)$ are distinct positive real numbers, and $\beta=$ $\prod_{i=1}^{n}\left(1+\alpha_{i}\right)$.

Now, for distinct positive $\alpha_{i}, M$ is a normal hyperbolic operator with the distinct characteristic sheets through a point $\left(x^{0}, y^{0}, z^{0}\right)$ of the form $\left(x-x^{0}\right)^{2}+\left(y-y^{0}\right)^{2}=\alpha_{i}\left(z-z^{0}\right)^{2}$. It is a result of I. G. Petrovsky (see [1]), that the Cauchy problem (1.22), (1.23) has the unique $C^{\infty}$ solution $\bar{V}(x, y, z)$ in that part $R_{\alpha}^{*}\left(\alpha=\left(\alpha_{1}, \alpha_{2}, \cdots, \alpha_{n}\right)\right)$ of the domain of influence of the initial surface $\sigma$ for the equation $M \bar{V}(x, y, z)=$ $-\beta \varphi(x, y,-z)$, which lies in $\bar{R}$, so that $\varphi(x, y,-z)$ is defined.

In view of the identity

$$
\prod_{i=1}^{n}\left(1+\alpha_{i}\right) \frac{\partial^{2 n}}{\partial z^{2 n}}-\prod_{i=1}^{n}\left[\frac{\partial^{2}}{\partial z^{2}}-\alpha_{i}\left(\frac{\partial^{2}}{\partial x^{2}}+\frac{\partial^{2}}{\partial y^{2}}\right)\right]=P(\Delta)
$$

where $P$ is a polynomial in $\partial / \partial x, \partial / \partial y, \partial / \partial z$ the function $V^{*}(x, y, z)$, which solves system (S) in $G^{\prime}$ satisfies the above Cauchy problem (1.22), (1.23) in the neighborhood of the initial surface $\sigma$, and by uniqueness, 
the solution $\bar{V}(x, y, z) \in R_{\alpha}^{*}$ must coincide with $V^{*}(x, y, z)$ in that neighborhood. Consequently, $\Delta \bar{V}$ and all its derivatives vanish on $\sigma$.

Since the operators $M$ and $\Delta$ commute, operating on equation (1.22) by $\Delta$ we obtain $M(\Delta \bar{V})=0$. Therefore, by uniqueness of the solution of Cauchy's problem for $M(\Delta \bar{V})=0$ with homogeneous initial conditions, we conclude that $\bar{V}(x, y, z)$, which solves (1.22), (1.23), is harmonic in $R_{a}^{*}$ and solves system (S) in this domain.

Putting $U(x, y, z)=\left(\partial^{2 n-1} / \partial z^{2 n-1}\right) \bar{V}(x, y, z)$ for $(x, y, z) \in R_{\alpha}^{*}$ we have constructed the harmonic extension of $U$ into $R \cup \sigma \cup R_{\alpha}^{*}$. We now observe, that as $\alpha_{i} \rightarrow 0(i=1,2, \cdots, n)$ the characteristic surfaces of $M$ close down on parallels to the $z$-axis. It follows, that every point of $\bar{R}$ is in some $R_{\alpha}^{*}$ for $\alpha_{i}$ sufficiently small. In view of the simple connectedness of $R \cup \sigma \cup \bar{R}$, the harmonic extension of $U$ at any point of $\bar{R}$ cannot depend on $\alpha$, and it follows that $U$ can be harmonically extended into all of $R \cup \sigma \cup \bar{R}$. Thus,

THeorem 1. If $U(x, y, z)$ is harmonic in $R, U \in C^{1}$ in $R \cup \partial R$, and satisfies condition (1.2) on $\sigma$, then $U$ can be harmonically extended into $R \cup \sigma \cup \bar{R}$.

REMARK The construction of the extension of $U$ depended on the solution of a hyperbolic problem whose order is twice the degree of the polynomial $P_{n}(x, y)$, the coefficient in the first order boundary condition. This illustrates the difficulty of extending our result to the case of, say, a coefficient $f(x, y)$, which is an entire function.

2. Extension of solutions of the wave equation. We consider an open domain $D:\{-m<x<0,-l<y<l,-l<t<l\}$ and the plane region $\sigma:\{x=0,-l<y<l,-l<t<l\}$. Denote, for any domain $\mathscr{D}$, the mirror image of $\mathscr{D}$ with respect to the $x=0$ plane by $\overline{\mathscr{D}}$.

Let there be given a real function $U(x, y, t), U \in C^{4}$ in the closure of $D$, such that:

$$
\begin{array}{rr}
L U \equiv U_{x x}+U_{y y}-U_{t t}=0 & \text { in } D \\
U_{x}+\alpha U_{y}+(A y+B) U=0 & \text { on } \sigma
\end{array}
$$

where $\alpha, A, B$ are real constants; $\alpha \neq 0$.

Define a function $V(x, y, t)$ for $(x, y, t) \in D \cup \sigma$ :

$$
V(x, y, t) \equiv \int_{0}^{x} U(\xi, y, t) d \xi+G(y, t)
$$

where $G(y, t)$ is the $C^{4}$ solution of the Cauchy problem:

$$
\left.\begin{array}{l}
G_{y y}-G_{t t}+U_{x}(0, y, t)=0 \\
G(y, 0)=G_{t}(y, 0)=0
\end{array}\right\}
$$


Let $P$ be the parallelepiped bounded by the planes $t \pm y= \pm l$, $x=0, x=-m$. Then, $V(x, y, t) \in C^{4}\left(V_{x} \in C^{4}\right)$ is defined in $P \cap D \cup \sigma$, and we have the relations:

$$
\begin{gathered}
L V=0 \quad \text { in } P \cap D \cup \sigma, \\
V_{x x}+\alpha V_{x y}+(A y+B) V_{x}=0 \quad \text { on } P \cap \sigma,
\end{gathered}
$$

which are easily verified.

We now define for $(x, y, t) \in P \cap D \cup \sigma$ the function:

$$
\varphi(x, y, t) \equiv V_{x x}+\alpha V_{x y}+A\left(y \frac{\partial}{\partial x}-x \frac{\partial}{0 y}\right) V+B V_{x} .
$$

Since the operators $L$ and $\{y(\partial / \partial x)-x(\partial / \partial y)\}$ commute, operating on both sides of (2.7) by $L$, and making use of (2.5), we obtain:

$$
L \varphi=\left\{\frac{\partial^{2}}{\partial x^{2}}+\alpha \frac{\partial^{2}}{\partial x \partial y}+(A y+B) \frac{\partial}{\partial x}-A x \frac{\partial}{\partial y}\right\}(L V)=0 .
$$

Setting $x=0$ in (2.7), and making use of (2.6) we have $\varphi(0, y, t)=0$.

If we now set for $(x, y, t) \in \bar{P} \cap \bar{D} \cup \sigma$

$$
\varphi(x, y, t)=-\varphi(-x, y, t)
$$

it follows, that $L \varphi=0$ in $P \cap D \cup \sigma \cup \bar{P} \cap \bar{D}$, and $\varphi \in C^{3}$.

Since $\varphi(-x, y, t)$ is known for $(x, y, t) \in \bar{P} \cap \bar{D} \cup \sigma$, we now seek a function $\bar{V}(x, y, t)$ for $(x, y, t) \in \bar{P} \cap \bar{D} \cup \sigma$, which solves the following Cauchy problem:

(2.8) $M \bar{V}(x, y, t) \equiv \bar{V}_{x x}+\alpha \bar{V}_{x y}+(A y+B) \bar{V}_{x}-A x \bar{V}_{y}=-\varphi(-x, y, t)$

$$
\bar{V}(0, y, t)=G(y, t), \quad \bar{V}_{x}(0, y, t)=U(0, y, t) \quad \text { on } \bar{P} \cap \sigma .
$$

It is well known, that the function $\bar{V}(x, y, t) \in C^{4}$, which satisfies (2.8), (2.9), exists in a domain $Q$. Here $Q$ is that domain, each of whose sections by a plane $t=K(-l<K<l)$ is a right triangle bounded by $x=0, y=l-|K|$ and $y-\alpha x=|K|-l$ if $\alpha>0$, or by $x=0, y=$ $|K|-l$ and $y-\alpha x=l-|K|$ if $\alpha<0$. Note that $Q$ does not depend on $U$, and is a subdomain of $\bar{P} \cap \bar{D} \cup \sigma$.

Lemma 4. If $\bar{V}(x, y, t) \in C^{4}$ in $Q$ is the solution of the Cauchy problem (2.8), (2.9), then $L \bar{V}=0$ in $Q$.

Proof. We operate on both sides of (2.8) by $L$. Since the operators $L$ and $\{y(\partial / \partial x)-x(\partial / \partial y)\}$ commute, and $L \varphi(-x, y, t)=0$, we obtain:

$$
M(L \bar{V})=0 \text {. }
$$


setting $x=0$ in (2.8) we have,

$$
\bar{V}_{x x}(0, y, t)=-\alpha \bar{V}_{x y}(0, y, t)-(A y+B) \bar{V}_{x}(0, y, t)
$$

and hence, making use of (2.9) and (2.2), we obtain:

$$
\bar{V}_{x x}(0, y, t)=U_{x}(0, y, t) \text {. }
$$

Thus, due to equations (2.9) and (2.4)

$$
\left.L \bar{V}\right|_{x=0}=0 \text {. }
$$

From (2.3) and (2.7) we have:

$$
\begin{array}{r}
\left.\varphi(-x, y, t) \equiv \varphi(\xi, y, t)\right|_{\xi=-x}=\left.U_{\xi}(\xi, y, t)\right|_{\xi=-x}+\alpha U_{y}(-x, y, t) \\
+(A y+B) U(-x, y, t)+A x G_{y}(y, t)+A x \int_{0}^{-x} U_{y}(\xi, y, t) d \xi
\end{array}
$$

and therefore,

$$
\begin{aligned}
\left.\frac{\partial}{\partial x} \varphi(-x, y, t)\right|_{x=0}= & -U_{x x}(0, y, t)-\alpha U_{x y}(0, y, t) \\
& -(A y+B) U_{x}(0, y, t)+A G_{y}(y, t) .
\end{aligned}
$$

Differentiating (2.8) with respect to $x$, and setting $x=0$ we obtain

$$
\bar{V}_{x x x}+\alpha \bar{V}_{x x y}+(A y+B) \bar{V}_{x x}-A \bar{V}_{y}=-\left.\frac{\partial}{\partial x} \varphi(-x, y, t)\right|_{x=0} \quad \text { on } x=0,
$$

which after substituting (2.9), (2.10) and (2.11) becomes:

$$
\bar{V}_{x x x}(0, y, t)=U_{x x}(0, y, t) \text {. }
$$

Hence, by (2.9) and (2.1),

$$
\left.\frac{\partial}{\partial x} L \bar{V}\right|_{x=0}=0
$$

Consequently, by uniqueness of the solution of Cauchy's problem for $M(L \bar{V})=0$ with homogeneous initial conditions, we have that $L \bar{V}=0$ in $Q$.

We thus have:

THeOREM 2. If $U(x, y, t) \in C^{4}$ in the closure of $D$ solves the wave equation (2.1) and satisfies the boundary condition (2.2) on $\sigma$, then there exists a function $U=\bar{V}_{x} \in C^{3}$ in the subdomain $Q$ of $\bar{D}$, which extends $U$ across $\sigma$ as $C^{3}$ solution of the wave equation. 


\section{REFERENCES}

1. L. Gårding, Solution directe du problème de Cauchy pours les équations hyperboliques, Colloques internationaux du Centre National de la Recherche Scientifique, LXXI, Nancy, 1956.

2. E. Hopf, Über den funktionalen, insbesondere den analytischen Charakter der Lösungen elliptischer Differential-gleichungen zweiter Ordnung, Math. Zeitschrift 34 (1932), 194-233.

3. H. Lewy, $A$ note on harmonic functions and a hydrodynamical application, Proc. Amer. Math. Soc., 3, no. 1, (1952).

UNIVERSITY OF CALIFORNIA, BERKELEY 



\section{PACIFIC JOURNAL OF MATHEMATICS}

\section{EDITORS}

\author{
Robert Osserman \\ Stanford University \\ Stanford, California
M. G. Arsove
University of Washington
Seattle 5 , Washington

\author{
J. DugundJI \\ University of Southern California \\ Los Angeles 7, California
}

Lowell J. Paige

University of California

Los Angeles 24, California

\section{ASSOCIATE EDITORS}
E. F. BECKENBACH
B. H. NeumanN
F. WOLF
K. YOSIDA

\section{SUPPORTING INSTITUTIONS}

\author{
UNIVERSITY OF BRITISH COLUMBIA \\ CALIFORNIA INSTITUTE OF TECHNOLOGY \\ UNIVERSITY OF CALIFORNIA \\ MONTANA STATE UNIVERSITY \\ UNIVERSITY OF NEVADA \\ NEW MEXICO STATE UNIVERSITY \\ OREGON STATE UNIVERSITY \\ UNIVERSITY OF OREGON \\ OSAKA UNIVERSITY \\ UNIVERSITY OF SOUTHERN CALIFORNIA
}

\author{
STANFORD UNIVERSITY \\ UNIVERSITY OF TOKYO \\ UNIVERSITY OF UTAH \\ WASHINGTON STATE UNIVERSITY \\ UNIVERSITY OF WASHINGTON \\ * * * * \\ AMERICAN MATHEMATICAL SOCIETY \\ CALIFORNIA RESEARCH CORPORATION \\ SPACE TECHNOLOGY LABORATORIES \\ NAVAL ORDNANCE TEST STATION
}

Mathematical papers intended for publication in the Pacific Journal of Mathematics should by typewritten (double spaced), and on submission, must be accompanied by a separate author's résumé. Manuscripts may be sent to any one of the four editors. All other communications to the editors should be addressed to the managing editor, L. J. Paige at the University of California, Los Angeles 24, California.

50 reprints per author of each article are furnished free of charge; additional copies may be obtained at cost in multiples of 50 .

The Pacific Journal of Mathematics is published quarterly, in March, June, September, and December. Effective with Volume 13 the price per volume (4 numbers) is $\$ 18.00$; single issues, $\$ 5.00$. Special price for current issues to individual faculty members of supporting institutions and to individual members of the American Mathematical Society: $\$ 8.00$ per volume; single issues $\$ 2.50$. Back numbers are available.

Subscriptions, orders for back numbers, and changes of address should be sent to Pacific Journal of Mathematics, 103 Highland Boulevard, Berkeley 8, California.

Printed at Kokusai Bunken Insatsusha (International Academic Printing Co., Ltd.), No. 6, 2-chome, Fujimi-cho, Chiyoda-ku, Tokyo, Japan.

PUBLISHED BY PACIFIC JOURNAL OF MATHEMATICS, A NON-PROFIT CORPORATION

The Supporting Institutions listed above contribute to the cost of publication of this Journal, but they are not owners or publishers and have no responsibility for its content or policies. 


\section{Pacific Journal of Mathematics}

\section{Vol. 14, No. $3 \quad$ July, 1964}

Erik Balslev and Theodore William Gamelin, The essential spectrum of a class of ordinary differential operators . . . . . . . . . . . . . . . . . . . .

James Henry Bramble and Lawrence Edward Payne, Bounds for derivatives in

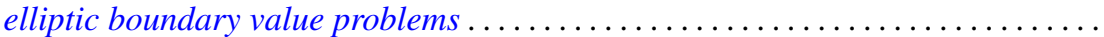

Hugh D. Brunk, Integral inequalities for functions with nondecreasing

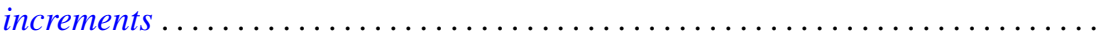

William Edward Christilles, A result concerning integral binary quadratic

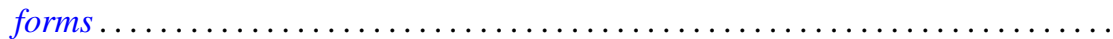

Peter Crawley and Bjarni Jónsson, Refinements for infinite direct decompositions of

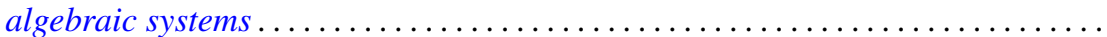

Don Deckard and Carl Mark Pearcy, On continuous matrix-valued functions on a Stonian space.

Raymond Frank Dickman, Leonard Rubin and P. M. Swingle, Another

characterization of the $n$-sphere and related results $\ldots \ldots \ldots \ldots \ldots \ldots$

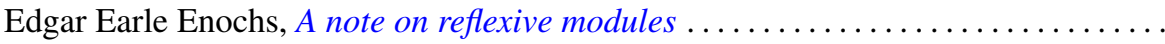

Vladimir Filippenko, On the reflection of harmonic functions and of solutions of the

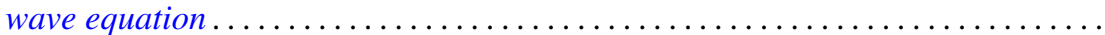

Derek Joseph Haggard Fuller, Mappings of bounded characteristic into arbitrary

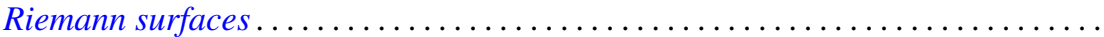
895

Curtis M. Fulton, Clifford vectors . . . . . . . . . . . . . . . . . . . . . . . . . . . . . . 917

Irving Leonard Glicksberg, Maximal algebras and a theorem of Radó . .

919

Kyong Taik Hahn, Minimum problems of Plateau type in the Bergman metric

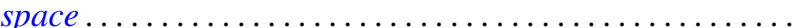

A. Hayes, A representation theory for a class of partially ordered rings...

J. M. C. Joshi, On a generalized Stieltjes trasform

J. M. C. Joshi, Inversion and representation theorems for a generalized Laplace transform ...

Eugene Kay McLachlan, Extremal elements of the convex cone $B_{n}$ of functions ...

Robert Alan Melter, Contributions to Boolean geometry of p-rings ...

James Ronald Retherford, Basic sequences and the Paley-Wiener criterion . . . . . . . 1019

Dallas W. Sasser, Quasi-positive operators. .

Oved Shisha, On the structure of infrapolynomials with prescribed coefficients ..

Oved Shisha and Gerald Thomas Cargo, On comparable means

Maurice Sion, A characterization of weak ${ }^{*}$ convergence ........

Morton Lincoln Slater and Robert James Thompson, A permanent inequality for

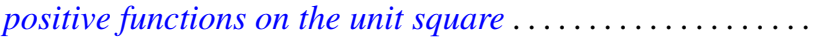

David A. Smith, On fixed points of automorphisms of classical Lie algebras ...

Sherman K. Stein, Homogeneous quasigroups ................

J. L. Walsh and Oved Shisha, On the location of the zeros of some infrapolynomials with prescribed coefficients .

Ronson Joseph Warne, Homomorphisms of $d$-simple inverse semigroups with identity . 